\title{
Lusotopie
}

Recherches politiques internationales sur les espaces

issus de l'histoire et de la colonisation portugaises

XVI(2) | 2009

Afrobrésiliennité ? Luso-afrobrésiliennité ?

\section{Le scrutin municipal d'octobre 2008 au Brésil}

Pour une analyse des résultats électoraux à l'aune des variations de l'offre politique

O escrutíno municipal de outubro de 2008 no Brasil. Para uma análise dos resultados eleitorais pelas variações da oferta política

The October 2008 Municipal Elections in Brazil. Analysis of the Election Results in the Light of Variations in the Political Offering

\section{Marie-Hélène Sá Vilas Boas}

\section{(2) OpenEdition}

\section{Journals}

Édition électronique

URL : http://journals.openedition.org/lusotopie/138

ISSN : 1768-3084

Éditeur :

Association des rechercheurs de la revue Lusotopie, Brill, Karthala

Édition imprimée

Date de publication : 1 juin 2009

Pagination : 11-25

ISSN : 1257-0273

Référence électronique

Marie-Hélène Sá Vilas Boas, « Le scrutin municipal d'octobre 2008 au Brésil », Lusotopie [En ligne], XVI

(2) | 2009, mis en ligne le 23 septembre 2015, consulté le 26 avril 2019. URL : http://

journals.openedition.org/lusotopie/138 


\section{LE SGRUTIN MUNICIPAL D'OCTOBRE 2008 AU BRÉSIL Pour une analyse des résultats électoraux à l'aune des variations de l'offre politique}

Les résultats des élections municipales sont généralement appréhendés, par les chercheurs brésiliens, à partir du score de chacun des partis politiques, sans toutefois qu'une telle démarche ne soit explicitée. L'objectif de cet article est d'interroger la pertinence d'un traitement, par parti politique, des résultats électoraux locaux au Brésil. À partir de l'étude des scrutins municipaux d'octobre 2008, il s'agit de montrer qu'une analyse «partisane » des résultats renseigne moins sur la progression des partis politiques auprès de l'électorat qu'elle ne révèle le caractère mouvant de l'offre électorale locale au Brésil.

O escrutíno municipal de outubro de 2008 no Brasil

Para uma análise dos resultados eleitorais pelas variações da oferta política

As eleições municipais são geralmente consideradas, pelos pesquisadores brasileiros, a partir dos resultados de cada partido político, sem que esse método seja justificado. O objetivo desse artigo é discutir a pertinencia de uma tratamento, por partido, dos resultados eleitorais no Brasil. A ánalise das eleições municipais de outubre de 2008 permite mostrar que o tratamento, "por partido », dos resultados eleitorais informa pouco sobre o posicionamento político do eleitorado mais revela o carater instavél da oferta eleitoral local.

The October 2008 Municipal Elections in Brazil

Analysis of the Election Results in the Light of Variations in the Political Offering

Municipal election results are generally analysed by Brazilian researchers on the basis of the score of each of the political parties, although this method is not explicitly stated. The purpose of this article is to analyse the pertinence of processing local election results in Brazil per political party. On the basis of a study of the October 2008 municipal elections, it is shown that a 'party' analysis of the results does not so much provide information about the progression of the political parties among the electorate as it reveals the shifting nature of the local political offering in Brazil.

"厂 e PMDB sera la fiancée la plus prisée par le gouvernement et l'opposition », titrait le journal $O$ Estado de São Paulo au lendemain du scrutin municipal d'octobre 2008'. En remportant le plus grand nombre de municipalités $(21,7 \%$, de votes nominaux des maires $(18,7 \%$ ) ainsi que deux nouvelles capitales, Rio de Janeiro et Porto Alegre, le très hétérogène $\mathrm{PMDB}^{2}$ a été unanimement présenté comme le «vainqueur» des scrutins de 2008 par la presse brésilienne. Un tel

${ }^{1}$ Voir ci-après le cadre des sigles des partis politiques brésiliens. Le « PMDB cresce e vira a noiva mais cobiçada por governo e oposição », Estadão, 27 octobre 2008.

${ }^{2}$ PMDB ( $c$. infra) est un parti généralement classé au centre de l'échiquier politique brésilien. Il regroupe des élus s’identifiant tant à la gauche qu'à la droite du champ politique. 
«verdict électoral», érigeant un parti politique, le PMDB, en acteur victorieux des convocations municipales s'est imposé au lendemain du scrutin et a constitué le point de départ des interprétations multiples et, parfois, contradictoires, énoncées par les médias et les acteurs politiques.

Le traitement, par partis politiques, des résultats municipaux, est également au cœur des études scientifiques réalisées sur les élections municipales. Ainsi des travaux de géographie électorale ${ }^{4}$, des comparaisons entre scrutins ${ }^{5}$ ainsi que des analyses écologiques du vote ${ }^{6}$, élaborés ces dernières années par des chercheurs des deux côtés de l'Atlantique. Ces analyses appréhendent généralement les évolutions électorales " par le bas ", c'est-à-dire à partir du sens que l'électeur confère à son vote, que ce dernier soit associé aux clivages partisans (les électeurs ont voté pour tel parti), idéologiques (un vote de droite/gauche, progressiste/conservateur) ou que les déterminants du vote soient considérés à partir d'enjeux locaux ou nationaux (un vote sanction/d'adhésion). Or, ce traitement des scrutins locaux, inscrit dans une philosophie politique de la représentation érigeant l'élection en moment privilégié d'expression des préférences des gouvernés, n'est que peu pertinent, en particulier au Brésil où le système partisan est fragmenté, peu institutionnalisé et mal connu de la majorité de l'électorat. Cette interprétation des résultats tend, en outre, à sous-estimer le fait que l'acte électoral constitue un échange politique dont les contours sont en partie maîtrisés par les entrepreneurs politiques ${ }^{7}$. Comme le rappelle Gaetano Mosca, «lorsque nous disons que les électeurs élisent leur député, nous nous exprimons sans aucune précision. La vérité est que le député se fait élire par ses électeurs $»^{8}$. Aussi, souhaitons-nous faire l'hypothèse, dans cet article, qu'une analyse partisane des résultats municipaux renseigne moins sur le sens que l'électorat confère au scrutin qu'elle ne révèle le caractère mouvant de l'offre électorale locale au Brésil. Plus précisément, l'on se demandera dans quelle mesure les variations électorales dépendent des usages que les entrepreneurs politiques locaux font du système de partis brésilien.

Les élections municipales d'octobre 2008 constituent, à cet égard, un cas particulièrement intéressant : ce que les observateurs ont nommé « victoire du PMDB » repose, en partie, sur l'élection d'entrepreneurs politiques locaux ayant rejoint cette formation à la veille du scrutin de 2008. En partie seulement car il ne s'agit pas, ainsi que le souligne Michel Offerlé, d'«abandonner l'idée naïve du citoyen générant le représenté pour la remplacer par l'idée cynique de l'entrepreneur manipulant le processus de représentation ${ }^{9} »$. L'ambition de cet article n'est, toutefois,

${ }^{3}$ Les résultats des élections municipales sont présentés en annexe 1.

${ }^{4}$ G.R. Jacob, D. Hees, P. Waniez \& V. Brustlein, « As Eleições municipais e sua influência nas disputas eleitorais ", Alceu (Rio de Janeiro), V, 2002 : 135-181.

${ }^{5}$ D.V. Fleischer, «As Eleições Municipais no Brasil : Uma análise comparativa (1982-2000) », Opinião Pública (São Paulo), VIII (1), 2002 : 80-105.

${ }^{6}$ L. Avelar \& M.I.M. Telles Walter, «Lentas mudanças o voto e a política tradicional », Opinião Pública (São Paulo), 14, 2008 : 102.

M. OfferLé, «Mobilisation électorale et invention du citoyen. L'exemple du milieu urbain à la fin du XIX ${ }^{\mathrm{e}}$ siècle », in D. Gaxie (ed.), Explication du vote. Un bilan des études électorales en France, Paris, Presses de la FNSP, 1989 ; A. GARrigou, Une histoire sociale du suffrage universel. 1848-2000, Paris, le Seuil, 2000 ( Point Histoire»).

${ }^{8}$ G. Mosca, Elementi di scienza politica, Turin, Fratelli Bocca, 1953, I : 206.

${ }^{9}$ M. OfFerLé, op. cit. : 161. 
pas de déterminer l'ensemble des facteurs explicatifs des variations électorales mais davantage de mettre en lumière un aspect peu abordé par l'analyse électorale brésilienne, c'est-à-dire la manière dont les variations de l'offre politique influent sur les résultats des scrutins municipaux.

Afin d'explorer notre hypothèse, nous nous appuierons sur les données publiées par le Tribunal supérieur électoral (TSE) depuis 2000, veille de l'arrivée au pouvoir de Luiz Inácio Lula da Silva à la Présidence de la République. Après avoir interrogé, dans un premier temps la pertinence d'une analyse "partisane » des scrutins municipaux au Brésil, nous montrerons que les résultats de 2008, et plus particulièrement la progression du $\mathrm{PMDB}$, dépendent en partie du caractère mouvant de l'offre électorale locale.

\section{Sigles des partis politiques brésiliens}

PAN, Partido dos Aposentados da Nação, Parti des retraités de la nation

PC do B, Partido Comunista do Brasil, Parti communiste du Brésil

PCB, Partido Comunista Brasileiro, Parti communiste brésilien

PCO, Partido da Causa Operária, Parti de la cause ouvrière

PDT, Partido Democrático Trabalhista, Parti démocrate-travailliste

PFL, Partido da Frente Liberal, Parti du front libéral

PHS, Partido Humanista da Solidariedade, Parti humaniste de la solidarité

PL, Partido Liberal, Parti libéral

PMDB, Partido do Movimento Democrático Brasileiro, Parti du mouvement démocratique brésilien

PMN, Partido da Mobilização Nacional, Parti de la mobilisation nationale

PP, Partido Progressista, Parti progressiste

PPS, Partido Popular Socialista, Parti populaire socialiste

PRB, Partido Republicano Brasileiro, Parti républicain brésilien

PRP, Partido Republicano Progressista, Parti républicain progressiste

Prona, Partido de Reedificação da Ordem Nacional, Parti de la réédification de l'ordre national

PRTB, Partido Renovador Trabalhista Brasileiro, Parti rénovateur travailliste brésilien

PSB, Partido Socialista Brasileiro, Parto socialiste brésilien

PSC, Partido Social Cristão, Parti social-chrétien

PSDB, Partido da Social Democracia Brasileira, Parti de la social-démocratie brésilienne

PSDC, Partido Social Democrata Cristão, Parti social-démocrate chrétien

PSL, Partido Social Liberal, Parti social-libéral

Psol, Partido Socialismo e Liberdade, Parti Socialisme et liberté

PSTU, Partido Socialista dos Trabalhadores Unificado, Parti socialiste unifié des travailleurs

PT, Partido dos Trabalhadores, Parti des travailleurs

PTB, Partido Trabalhista Brasileiro, Parti travailliste brésilien

PTC, Partido Trabalhista Cristão, Parti travailliste chrétien

PT do B, Partido Trabalhista do Brasil, Parti travailliste du Brésil

PTN, Partido Trabalhista Nacional, Parti travailliste national

PV, Partido Verde, Parti vert 


\section{Quelle pertinence pour un traitement " partisan " des résultats?}

Les résultats électoraux sont appréhendés, par la presse et certains analystes, à partir des scores réalisés par chacun des partis politiques. Ce traitement des résultats ne va toutefois pas de soi dans le contexte brésilien. En effet, bien que la loi électorale impose, à tout candidat à l'élection, de se présenter sous une étiquette partisane, le système de parti est faiblement institutionnalisé et ne structure que peu les choix électoraux. Aussi, un traitement des résultats par organisation partisane ne rend que peu compte de la progression des formations politiques au sein de l'électorat, ce que le scrutin de 2008, et plus particulièrement la progression des résultats du PMDB, permettent d'illustrer.

\section{La faible institutionnalisation du système de partis brésilien}

Le traitement des résultats par partis présente plusieurs limites en raison du type de scrutin étudié ainsi que des caractéristiques du système politique brésilien.

Plusieurs auteurs soulignent en effet que, dans le cas français, si l'on additionne le nombre de voix et de municipalités remportées par les organisations partisanes, le traitement des données électorales par formations tend à rendre homogène une offre politique dont les déclinaisons locales peuvent être très diversifiées ${ }^{10}$. De même, cette approche unifie le sens de votes souvent motivés par des déterminants variables.

Les limites d'une approche "partisane » sont d'autant plus importantes, dans le cas brésilien, que les partis politiques constituent des acteurs peu structurants de la vie politique brésilienne, faiblesse en partie attribuée au mode de scrutin. Au Brésil, l'exécutif municipal est élu au scrutin uninominal à un tour pour les villes de moins de 200000 habitants et à deux tours pour les villes de plus de 200000 habitants. Les électeurs sont invités tous les quatre ans à choisir entre les divers «tickets» présentés par les partis ou les coalitions partisanes afin d'élire le maire (prefeito) et le vice-maire (vice-prefeito). Le législatif est, quant à lui, élu au scrutin proportionnel de liste ouverte, les électeurs ayant la possibilité de choisir leur candidat parmi les listes électorales ou de donner leur voix à un parti (voto de legenda).

Le système de liste ouverte alimente une forte concurrence entre candidats de listes concurrentes mais également au sein d'une même liste ${ }^{11}$. Les stratégies de campagnes des candidats sont généralement centrées sur l'individu et moins sur l'organisation partisane, les votos de legenda représentant un faible pourcentage des voix $^{12}$. La personnalisation des campagnes n'est, toutefois, pas un phénomène homogène et varie selon les formations. Ainsi, David Samuels montre que durant

${ }^{10}$ P. Lehingue, «Mais qui a gagné ? Les mécanismes de production des verdicts électoraux », in J. Lagroye, P. Lehingue, \& F. Sawicki (eds), Mobilisations électorales. Le cas des élections municipales de 2001, Paris, PUF, 2005 : 323-358.

${ }^{11}$ Cf. Le chapitre 7 de l'ouvrage de D. Vidal, La politique au quartier, Paris, Éditions de la MSH, 1998 : 173-212.

12 D. SAmuels, «Determinantes do Voto Partidário em Sistemas Eleitorais Centrados no Candidato: Evidências sobre o Brasil », Dados (Rio de Janeiro), XL (3), 1997 : 340-365. 
les années 1990, les votes de legendas constituaient une part non négligeable des résultats du PT et de la petite formation de droite le Prona ${ }^{13}$.

La répartition proportionnelle des sièges, pour l'élection du législatif, ne dépend pas de l'obtention d'un seuil minimal de voix, par partis, favorisant ainsi une fragmentation du système partisan. On comptait, en 2008, 37 partis politiques ayant participé à l'élection, sur l'ensemble du territoire brésilien. La combinaison entre proportionnalité du scrutin et système partisan fragmenté alimente, enfin, la conclusion d'alliances, entre formations partisanes, durant la campagne (les coligações eletorais) pour la définition des «tickets» et des listes électorales, alliances bien souvent redéfinies au lendemain de l'élection, lorsque sont déterminés les gouvernements de coalition.

La complexité du système électoral explique, selon Maria Kinzo, que le système partisan ne structure que peu le choix des électeurs et que ces derniers ne déclarent que rarement s'identifier à une organisation partisane ${ }^{14}$ avec, de nouveau, des variations selon les formations. Si le PT et le PMDB suscitaient, sur la période 1989-2002, l'«identification» de 10 à $16 \%$ des personnes interrogées par l'institut Datafolha, d'autres partis d'envergure nationale, tels que le PSDB ou le PFL ne suscitaient que 1 à $5 \%$ d'identification pour le premier et 2 à $6 \%$ pour le second ${ }^{15}$.

Le constat de "victoire d'un parti politique » s'avère ainsi peu pertinent au regard de ces caractéristiques, s'il est interprété comme une progression du parti au sein de l'électorat. Le scrutin de 2008 l'illustre puisque la progression proclamée du PMDB correspond principalement à une agrégation de victoires individualisées et localisées dont on peut questionner le caractère "partisan ».

\section{La "victoire du PMDB ": l'agrégation d'élections individualisées et localisées}

Au lendemain de l'élection, les médias et les acteurs politiques se sont livrés au traditionnel jeu de désignation des vainqueurs et des vaincus. Alors que le PMDB a été unanimement proclamé victorieux, le PT, le PSDB et le DEM (anciennement PFL) ont été parfois présenté en recul ou en progression selon les lignes éditoriales et des données mobilisées à l'appui de l'argumentation. Le constat de victoire du PMDB a ainsi consolidé une interprétation du scrutin centrée sur les acteurs partisans. Or, l'on peut se demander dans quelle mesure cette lecture rend compte des dynamiques plurielles de l'élection municipale.

${ }^{13}$ Par exemple, lors des élections présidentielles de 1994, la candidature de Lula recueillit $24,04 \%$ des voix dont $33 \%$ de votos de legendas. La petite formation de droite, le Prona, reçu cette même année, pour son candidat Eneas, 7,38\% de voix dont $81,7 \%$ de votes partisans, D. Samuels, op. cit.

${ }^{14}$ Selon Maria Kinzo, «les électeurs ont des difficultés à identifier les partis comme des acteurs politiques distincts c'est-à-dire comme des acteurs qui structurent le choix électoral et créent une identification. M. d'Alva Gil KInzo, «Os partidos no eleitorado: percepções públicas e laços partidários no Brasil », Revista brasileira de Cencias Sociais (São Paulo), 2005, XX (57): 65.

${ }^{15}$ Selon un sondage Datafolha du 7 octobre 2002, cité par M. d'Alva GIL Kinzo, op. cit.; L.F. VeIGA, «Os partidos brasileiros na perspectiva dos eleitores: mudanças e continuidades na identificação partidária e na avaliação das principais legendas após 2002 », Opinião Publica, XIII (2), $2007: 69$. 
En effet, ce «verdict électoral », qu'il s'agit, selon Patrick Lehingue, d'interpréter comme partie intégrante de la campagne ${ }^{16}$, a été construit à partir de l'agrégation de trois types de données: le nombre de mairies conquises par chacun des partis, les capitales gouvernées ainsi que le nombre de voix remportées par les candidats aux mandats exécutifs et législatifs. Or, chacune de ces entrées n'a pas la même portée analytique; elles ne peuvent être simplement additionnées pour désigner les vainqueurs et les vaincus.

Selon Jairo Nicolau, si l'on souhaite évaluer la progression des partis politiques au sein de l'électorat, l'indicateur le plus représentatif, bien que très imparfait, est le nombre de voix accordées aux conseillers municipaux, puisque celui-ci comprend les votos de legendas ${ }^{17}$. Or, cet indicateur atteste d'une grande stabilité des résultats par comparaison au scrutin de 2004, puisqu'à l'exception du DEM dont les scores sont en baisse de 1,5 point, les résultats du PT, du PMDB et du PSDB restent relativement constants.

Tabl. I. - Votes Nominaux ET « PARTisAns » POUR LES CONSEILlers MUNicipaux par parti, en pourcentage et valeur absolue (1 $1^{\text {er }}$ tour, 2000-2008)

\begin{tabular}{lrrrrrr}
\hline \multirow{2}{*}{ Partis } & \multicolumn{2}{c}{2000} & \multicolumn{2}{c}{2004} & \multicolumn{2}{c}{2008} \\
\cline { 2 - 7 } & \multicolumn{1}{c}{ Nb de voix } & \% des voix & Nb de voix & \% des voix & Nb de voix & \% des voix \\
\hline PDT & 5864359 & 6,7 & 6124376 & 6,3 & 6732459 & 6,5 \\
PFL/DEM & 10392045 & 12,0 & 9203801 & 9,4 & 7998671 & 7,7 \\
PL & 4418015 & 5,1 & 6121036 & 6,2 & 5444058 & 5,2 \\
PP & 7334209 & 7,5 & 7930516 & 9,1 & 7251598 & 7,0 \\
PMDB & 11588047 & 13,4 & 11061855 & 11,3 & 11968135 & 11,6 \\
PSDB & 9867430 & 11,4 & 10947044 & 11,2 & 10701758 & 10,4 \\
PSB & 4478449 & 4,6 & 3636150 & 4,2 & 5994659 & 5,8 \\
PT & 8132685 & 9,3 & 10431898 & 10,7 & 10517194 & 10,2 \\
PTB & 6893295 & 7,9 & 6844873 & 7,0 & 6319936 & 6,1 \\
\hline
\end{tabular}

Sources: Site du Tribunal supérieur électoral, <www.tse.gov.br>.

Les principales évolutions électorales, par comparaison avec le scrutin de 2004, concernent le nombre de voix remportées par les candidats à l'exécutif ainsi que le nombre de capitales "gagnées» par les candidats de chacun des partis. En effet, le PMDB a recueilli, en 2008, 18,4\% des votes nominaux des maires. Il progresse ainsi de plus de 3,5 points par rapport à 2004 et ravit la «première place » attribuée au PT, au lendemain de l'élection de 2004. Ce dernier a reçu, en effet, en 2008, 16,4\% des voix contre 17,1 en 2004.

${ }^{16}$ P. Lehingue, op. cit.

${ }^{17} \mathrm{~J}$. Nicolau, "Como avaliar quem saiu vencedor nas eleições municipais? », Veja (Brésil), 16 octobre 2008. Article consultable à l'adresse suivante: <http://veja.abril.com.br/politica/ blogs/eleicoes-2008/120042_comentarios.html>. 
Tabl. II. - Votes NOMINAUX DES MAIREs, PAR PARTI, AU PREMIER TOUR en valeur absolue et en pourcentage (2000-2008)

\begin{tabular}{lrrrrrr}
\hline \multirow{2}{*}{ Partis } & \multicolumn{2}{c}{2000} & \multicolumn{2}{c}{2004} & \multicolumn{2}{c}{2008} \\
\cline { 2 - 7 } & Nb de voix & \% des voix & Nb de voix & \% des voix & Nb de Voix & \% des voix \\
\hline PDT & 612065 & 6,6 & 6103294 & 6,4 & 6095495 & 6,1 \\
PFL /DEM & 12958862 & 15,3 & 11247438 & 11,8 & 9317989 & 9,0 \\
PL/ PR & 5612065 & 6,6 & 5022323 & 5,2 & 4274967 & 4,3 \\
PP & 6812860 & 8,0 & 6103294 & 6,4 & 6144206 & 6,1 \\
PMDB & 14249339 & 15,7 & 13258521 & 14,7 & 18479511 & 18,4 \\
PSDB & 13507709 & 16,0 & 15735933 & 16,5 & 14445415 & 14,4 \\
PSB & 3506432 & 4,2 & 4475295 & 4,7 & 5685266 & 5,7 \\
PT & 11938803 & 14,1 & 16326047 & 17,1 & 16529237 & 16,4 \\
PTB & 5803558 & 6,8 & 5255238 & 5,5 & 5066999 & 5,0 \\
\hline
\end{tabular}

Sources: ibid.

L'évolution des votes nominaux des maires pmedistes $^{18}$ repose, en grande partie, sur l'élection de candidats investis par cette même formation dans deux nouvelles capitales, Rio de Janeiro et Porto Alegre. Six capitales sont dorénavant gouvernées par des maires pmdébistes ${ }^{19}$, à l'égal du $\mathrm{PT}^{20}$. Or, il convient de s'arrêter quelque peu sur la portée de ces résultats. La conquête de Porto Alegre a été assurée par la reconduction du maire sortant José Fogaça, élu sous l'étiquette du PPS en 2004 et investi par le PMDB à la veille de l'élection de 2008. À Rio de Janeiro, le PMDB s'impose avec la victoire du très changeant Eduardo Paes, lequel avait auparavant été élu sous le sigle du PV, PFL, PTB, PSDB et, en 2008, du PMDB. Aussi, ce que la presse a nommé «victoire du PMDB» nous semble moins correspondre à la victoire d'un parti qu'à l'agrégation de victoires de personnalités locales ayant rejoint le PMDB pour le scrutin de 2008, alors que ce dernier constituait une ressource électorale particulièrement attractive pour les candidats locaux.

\section{Une offre électorale locale instable}

Le système partisan brésilien est complexe et instable : composé de trente-sept partis, dont une dizaine de petites formations sont renommées au gré des élections, il est également caractérisé par des vagues de «migration partisane », c'est-à-dire

${ }^{18}$ Relatif au PMDB.

${ }^{19}$ En plus de Rio de Janeiro et Porto Alegre, les quatre maires sortants du PMDB ont été réélus : il s'agit de Nelsinho Trad à Campo Grande (MS) et Iris Rezende à Goiania (GO), José Fogaça à Porto Alegre (RS), João Pinheiro à Salvador (BA), Diario Berger à Florianopolis (SG).

${ }^{20}$ Le PT a assisté à la reconduction de ses cinq maires sortants, à Vitória (ES) Rio Branco (AC), Fortaleza (CE) Raul Palmas (TO) et Porto Velho (RO.). Recife reste également aux mains de ce parti avec l'élection de João Paulo. Il occupe également la vice-présidence des gouvernements municipaux de Belo Horizonte et Aracaju. Cf. annexe 2. 
de changement de partis des élus, en particulier à la veille et au lendemain des consultations électorales ${ }^{21}$. Les sigles partisans font ainsi l'objet d'usages variés par les entrepreneurs politiques selon la ressource électorale qu'il représente. En 2008, le sigle pmdébiste semble avoir été particulièrement attractif et rétributeur pour les candidats à l'élection municipale.

\section{Les usages variés des étiquettes partisanes par les entrepreneurs politiques locaux}

Dans leur très grande majorité, les partis politiques brésiliens ne sont pas implantés sur l'ensemble du vaste territoire brésilien. Alors que certains ont une existence principalement régionale, tel que le PSB ou le PDT, d'autres partis d'envergure nationale pourtant, investissent des candidats dans certaines parties du territoire, tout en étant absent de la compétition électorale dans de nombreuses municipalités. Le nombre de candidatures, en particulier au poste de maires, présenté par chacun des partis dépend de la trajectoire propre à chacune des formations, de leur structuration interne mais également de la place qu'occupent ces formations dans les gouvernements estaduais et national. Certains partis sont, en effet, plus ou moins investis par les candidats, à la veille et au lendemain des élections, en fonction de la ressource électorale qu'ils représentent. Selon Simone Diniz, le phénomène de «migrations partisanes » est motivé par le "gain potentiel obtenu » que le changement d'organisation confère au candidat ${ }^{22}$. On observe, par exemple, au lendemain de l'élection de Lula, une migration d'élus vers le PT, laquelle explique en partie, selon Lucia Avelar et Maria Walter, la progression de cette formation dans certaines villes moyennes, lors des élections municipales de $2004^{23}$. L'alternance, à l'échelle nationale et estadual, s'accompagne selon ces deux auteurs, d'une redéfinition de la composition locale des partis politiques ${ }^{24}$ ainsi que de la ressource électorale que représente chacune des étiquettes partisanes. Les entrepreneurs politiques locaux font, ainsi, des usages variés des étiquettes partisanes, en particulier des formations caractérisées par une structuration lâche et un recrutement exogène ${ }^{25}$ parmi lesquels on compte le $\mathrm{PMDB}^{26}$.

Aussi est-il intéressant de considérer l'évolution du nombre de candidats présentés par les principaux partis au poste de maire, puisque celle-ci nous renseigne sur la capacité des formations à investir des candidats sur le territoire, laquelle ne dépend pas simplement de facteurs endogènes aux partis, comme nous venons de l'évoquer mais également de la place des partis dans les gouvernements des différents échelons de la fédération.

${ }^{21}$ Sur la «mobilité partisane », cf. A.M. SAntos, "Sedimentação de lealdades partidárias no Brasil : tendências e descompassos », Revista brasileira de Cienciais Sociais (São Paulo), 2001, XVI (45) : 69-83.

${ }^{22}$ S. Dinız, «As migrações partidárias e o calendário eleitoral », Revista Sociologia e Política (Curitiba), 15, novembre 2000: 31-48.

${ }^{23}$ L. Avelar \& M.I.M. Telles Walter, op. cit.

${ }^{24}$ Une vague de changement de partis d'élus fut, en effet, observable au lendemain de la démocratisation du régime, lorsque le PMDB accueillît des membres de l'UDN et du PDS.

${ }^{25}$ A.M. Santos, op. cit.

${ }^{26}$ S. Diniz, op. cit. 
Tabl. III. - Pourcentage de candidats au poste de maire et de maires Élus par parti politique (2000-2008)

\begin{tabular}{|c|c|c|c|c|c|c|c|c|c|}
\hline \multirow[t]{2}{*}{ Partis } & \multicolumn{3}{|c|}{2000} & \multicolumn{3}{|c|}{2004} & \multicolumn{3}{|c|}{2008} \\
\hline & $\begin{array}{c}\text { Candidats } \\
\text { (1) }\end{array}$ & $\begin{array}{l}\text { Élus } \\
\text { (2) }\end{array}$ & $(2) /(1)$ & $\begin{array}{l}\text { Candidats } \\
\text { (1) }\end{array}$ & $\begin{array}{c}\text { Élus } \\
\text { (2) }\end{array}$ & $(2) /(1)$ & $\begin{array}{c}\text { Candidats } \\
\text { (1) }\end{array}$ & $\begin{array}{l}\text { Élus } \\
\text { (2) }\end{array}$ & $(2) /(1)$ \\
\hline PDT & 17,1 & 5,2 & 31,9 & 15,3 & 5,4 & 35,5 & 17,66 & 6,3 & 37,4 \\
\hline PFL/DEM & 43,2 & 18,5 & 44,7 & 31,5 & 14,2 & 45,0 & 22,2 & 9,9 & 41,1 \\
\hline $\mathrm{PL} / \mathrm{PR}$ & 11,4 & 4,2 & 38,9 & 18,6 & 6,6 & 36,8 & 16,4 & 6,9 & 38,8 \\
\hline PP & 26,7 & 11,1 & 43,9 & 23,6 & 10,1 & 43,3 & 18,5 & 9,9 & 47,9 \\
\hline PMDB & 54,0 & 22,6 & 44,3 & 44,5 & 19,0 & 42,4 & 48,7 & 21,7 & 50,5 \\
\hline PSDB & 39,5 & 17,8 & 47,7 & 34,4 & 15,6 & 45,1 & 32,1 & 14,0 & 44,1 \\
\hline PSB & 8,6 & 2,4 & 27,7 & 11,1 & 3,0 & 28,5 & 15,8 & 5,6 & 27,5 \\
\hline PT & 24,9 & 3,3 & 14,2 & 35,1 & 7,4 & 21,2 & 29,4 & 9,9 & 20,5 \\
\hline
\end{tabular}

Source: Ibid.

En 2004, les partis du Président et du Vice-président de la République, le PT et le PL (aujourd'hui PR,) connaissaient une hausse sensible du nombre de leurs candidatures sur l'ensemble du territoire, puisque le premier augmentait de 11 points le nombre de ses compétiteurs $(35,1 \%$ de candidats en 2008 contre 24,9\% en $2000)$ et le second de 7 points (18,6\% en 2008 contre 11,4 en 2000). Le PMDB, à l'inverse, n'investissait que $44,5 \%$ de candidats soit une baisse de plus de 10 points par rapport à 2000 .

En 2008, le PMDB et certains petits partis d'implantation régionale, tels que le PSB et le PDT, investissent davantage de compétiteurs. Le PMDB passe, en effet, de 44,5\% de candidats au poste de maire en 2004 à 48,7 \% en 2008. Ses candidats sont, en outre, davantage élus puisque 50,5\% accèdent au poste de maire. Ce chiffre permet de renforcer l'hypothèse selon laquelle la progression du PMDB repose sur l'entrée d'élus locaux, à la veille du scrutin. En effet, les élections de 2008 sont caractérisées par un fort taux de reconduction des exécutifs municipaux puisque $67 \%$ des maires sortants ont été reconduits. Ces taux atteignent parfois $70 \%$ dans les États du Nord et du Nordeste ${ }^{27}$. Or, c'est principalement dans ces deux régions que l'on observe les principales variations de candidatures du PMDB en 2008.

La progression régionale des candidatures des principaux partis est, en outre, corrélée aux résultats électoraux. Le PMDB bénéficie d'une sensible progression du nombre de ses maires élus dans le Nord alors qu'il était surtout influent, en 2000, dans le Sud, le Sudeste et le Centre-Ouest. Les résultats du PT augmentent également dans ces mêmes régions depuis le début des années 2000, alors que ce parti était auparavant principalement implanté dans le sud du pays. Enfin, les résultats du DEM régressent sensiblement, en particulier dans le Nordeste, son principal bastion électoral.

${ }^{27}$ Le taux de reconduction des maires sortants s'élève à $75 \%$ dans l'État du Ceará, 74,3\% dans la Paraíba et 73,5\% dans l'Acre, Cf. «Taxa de reeleição em 2008 é a mais elevada da historia ", Folha de São Paulo, 10 octobre 2008. 


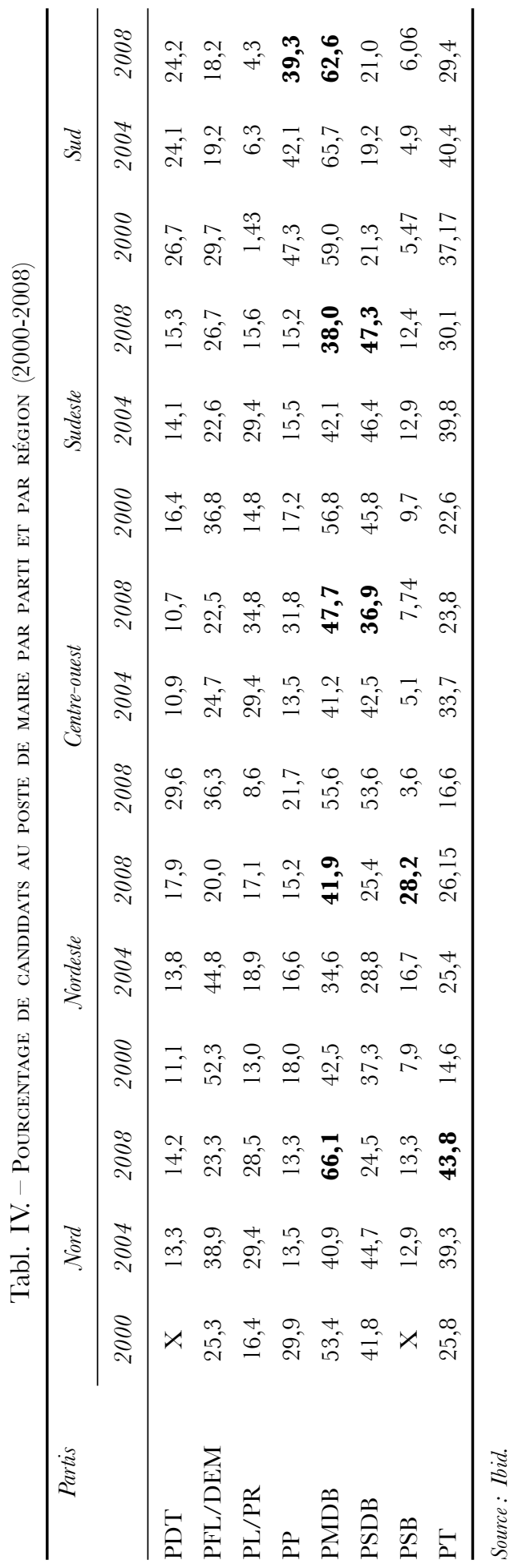


Tabl. V. - Pourcentage de maires élus par parti et par RÉgion (2000-2008)

\begin{tabular}{lcrrrrr}
\hline & Année & Nord & Nordeste & Sudeste & Sud & Centre-Ouest \\
\hline PMDB & 2000 & 18,2 & 19,9 & 22,5 & 28,1 & 23,6 \\
& 2004 & 13,8 & 14,9 & 16,8 & 31,4 & 14,8 \\
\multirow{3}{*}{ PFL-DEM } & 2008 & 26,7 & 18,6 & 14,5 & 32,5 & 22,7 \\
& 2000 & 21,6 & 24,9 & 13,3 & 14,9 & 18,5 \\
& 2004 & 9,4 & 23,1 & 21,1 & 7,2 & 7,7 \\
PSDB & 2008 & 8,6 & 8,3 & 11,0 & 6,5 & 8,8 \\
& 2000 & 19,6 & 16,1 & 20,7 & 10,9 & 29,9 \\
& 2004 & 19,6 & 12,9 & 20,9 & 7,7 & 21,7 \\
PT & 2008 & 10,2 & 10,7 & 23,2 & 7,8 & 14,8 \\
& 2000 & 4,7 & 1,2 & 4,9 & 3,0 & 0,6 \\
& 2004 & 12,9 & 3,5 & 8,9 & 7,9 & 7,5 \\
& 2008 & 14,4 & 7,5 & 11,0 & 10,6 & 8,6 \\
\hline
\end{tabular}

Source : Ibid.

Ainsi, l'étiquette pmdébiste semble avoir constitué une ressource particulièrement attractive pour les entrepreneurs locaux, dont il convient à présent d'analyser les causes.

\section{Le sigle Pmdebiste : une ressource électorale en 2008}

L'évolution des candidatures et des résultats du PMDB, en particulier dans le nord du pays, est un processus difficile à expliquer à partir des seuls résultats électoraux. On peut toutefois faire l'hypothèse que la position centrale qu'occupe ce parti dans le gouvernement de coalition nationale et à l'échelle estadual ${ }^{28}$, puisqu'il gouverne six États de la fédération, confère à ce parti un rôle pivot dans les jeux politiques et administratifs entre les divers échelons de la fédération, à l'origine d'un "gain potentiel important», pour reprendre les termes de Simone Diniz, pour ses élus. Le processus de «recentralisation de la fédération » suscité par l'introduction de politiques sociales ${ }^{29}$, parmi lesquelles le programme Bolsa Familia, a accru l'influence du gouvernement national sur l'action publique municipale. Ce même programme, dont la popularité explique en partie la réélection de Lula ${ }^{30}$, constitue également une ressource électorale pour les élus municipaux puisque l'application de cette politique est du ressort des municipalités, permettant ainsi aux maires d'en revendiquer les fruits. Or, la proximité partisane ou politique entre élus nationaux et locaux facilite les mécanismes de transferts financiers entre

${ }^{28}$ Relatif aux États fédérés. Nous conservons le terme brésilien estadual (estaduais au pluriel) pour éviter des confusions avec l'échelon national.

${ }_{29}$ M.H. Tavares de AlmeidA, «Recentralizando a federaçã ? ", Revista de Sociologia e Política, 24, $2005: 29-40$.

${ }^{30}$ G.A. Dillon Soares \& S.L. Terron, «Dois Lulas : a geografia eleitoral da reeleição (explorando conceitos, métodos e técnicas de análise geoespacial)», Opinião Publica, XIV (2), 2008: 269-301. 
divers niveaux de gouvernements, en particulier dans les États les plus dépendants de la fédération, c'est-à-dire dans le nord du pays ${ }^{31}$. Aussi peut-on supposer que la multi-positionnalité du PMDB, en facilitant l'accès aux ressources estaduais et fédérales, a attiré des acteurs politiques se présentant habituellement sous la bannière d'autres partis, notamment conservateurs. Le déclin des compétiteurs présentés par l'ancien PFL (aujourd'hui DEM), peut ainsi avoir alimenté le rang des candidats du très hétérogène $\mathrm{PMDB}$. Bien entendu, les processus de migrations partisanes reposent également sur une proximité idéologique ou organisationnelle entre formations partisanes, du moins pour les principaux partis. La forte hétérogénéité programmatique du PMDB et la diversité du profil de ses cadres partisans alimentent l'hypothèse selon laquelle ce parti a été le principal bénéficiaire de la mobilité des élus locaux. Cette formation "arc-en-ciel», selon l'expression de David Fleischer ${ }^{32}$, regroupe, en effet, des élus s'identifiant tant à la gauche qu'à la droite du champ politique brésilien ${ }^{33}$, à l'origine d'une malléabilité programmatique facilitant des stratégies d'alliances locales très variables. Le PMDB intègre, en effet, des coalitions à géométrie variable, composées de formations de l'ensemble de l'échiquier politique. La composition sociale de ce parti, généralement classé au centre du paysage politique, est, enfin, relativement semblable à celle de partis de «droite», tel que l'ancien PFL, puisqu'il est investi par une majorité de professions libérales, de chefs d'entreprises mais également de fonctionnaires ${ }^{34}$. Cette composition sociale est expliquée, par André Marenco et Miguel Serna, par la forte présence d'hommes politiques conservateurs dans le PMDB ${ }^{35}$.

Les cas d'Eduardo Paes et de José Fogaça, que nous avons déjà mentionnés, sont enfin, illustrateurs des processus migratoires dont a, sans doute, bénéficié le PMDB, de la part d'élu de «droite» et de la «gauche» du champ politique.

$* * *$

L'ambition de cet article était double: il s'agissait, dans un premier temps, de questionner le traitement, par partis, des résultats municipaux dans le cas brésilien. Alors qu'elle est bien souvent au cœur des commentaires médiatiques et scientifiques, cette approche n'est que peu explicitée et repose généralement sur une série de présupposés, en particulier de concordance entre les clivages du système partisan et les déterminants du vote de l'électorat. Or, le scrutin de 2008, et plus précisément le caractère localisé des victoires du PMDB, attestent des limites d'une association entre les résultats des partis politiques et le sens que l'électorat confère au scrutin. Par conséquent, nous avons préféré aborder les résultats à partir de l'offre électorale en nous demandant si les variations des résultats des formations

${ }^{31}$ M. Mendes \& G.A. Amorim Rocha, O que reelege um prefeito? Brasilia, Senado Federal, Rapport d'expertise, avril 2004.

32 D. Fleischer, «Elecciones Municipales Brasil, 3 de outubre de 1996 », Boletín Electoral Latinoamericano, XVI, San José de Costa Rica, IIDH-CAPEL, 1997 : 11-36.

33 C.E. Guzmán Mendoza \& E. Sena de Oliverra, « Brasil », in M. Alcántara \& F. Freindenberg (eds), Partidos Politicos de América Latina, Cono Sur, Salamanca, Universidad de Salamanca, IFE, 2001.

34 A. Marenco \& M. Serna, «Por que carreiras políticas na esquerda e na direita não são iguais ? Recrutamento legislativo em Brasil, Chile et Uruguai », Revista brasileira de Ciencias Sociais, XXII (64), juin 2007 : 93-113.

35 A. Marenco \& M. Serna, op. cit. : 110. 
politiques dépendent de la manière dont les organisations partisanes investissent et sont investies par les entrepreneurs politiques locaux. L'analyse du scrutin de 2008 nous a ainsi permis d'explorer l'hypothèse selon laquelle la progression des résultats du PMDB est liée à l'entrée d'élus locaux dans cette même formation, en raison de la ressource électorale que constituait l'étiquette pmdebiste. Cette analyse permet ainsi de confirmer qu'il existe des mécanismes de coordination entre systèmes partisans des divers échelons de la fédération ${ }^{36}$ : l'attractivité du PMDB reposent, en effet, sur le rôle pivot qu'il occupe dans les gouvernements de coalitions estaduais et national. Toutefois notre hypothèse ne peut être pleinement validée par la seule analyse des résultats municipaux et supposerait la réalisation d'un travail plus ample reposant sur des données plus précises. Une étude des déterminants des variations de l'offre électorale municipale et de son impact sur les scrutins municipaux constituerait, ainsi, un axe de recherche particulièrement fécond pour approfondir la connaissance de la vie politique locale brésilienne ${ }^{37}$.

Marie-Hélène SÁ VILAS BOAS IEP d'Aix-en-Provence et IEP de Lyon

${ }^{36}$ L. Piquet Carneiro \& M.H. Tavares de Almeida, «Defining the local political arena: municipal party systems in the brazilian federation », Dados, 2008, LI (2) : 403-432.

${ }^{37}$ Je remercie Denis Barbet ainsi que le comité de lecture de Lusotopie pour leurs conseils lors de la rédaction de cet article. Mes remerciements également à Sümbül Kaya pour la relecture de ce manuscrit. 


\section{Annexes}

Annexe I. - Nombre de municipalités et de votes nominaux des MAIRES REMPORTÉS par parti lors du premier tour d'octobre 2008

\begin{tabular}{|c|c|c|c|c|}
\hline & $\begin{array}{l}\text { Nombre de munici- } \\
\text { palités remportées }\end{array}$ & $\begin{array}{l}\% \text { du total des } \\
\text { municipalités }\end{array}$ & Votes nominaux & $\%$ du total des voix \\
\hline DEM & 493 & 8,89 & 9317989 & 9,3 \\
\hline PG do B & 41 & 3,4 & 1767500 & 1,7 \\
\hline PCB & 0 & 0,0 & 63785 & 0,1 \\
\hline $\mathrm{PCO}$ & 0 & 0,0 & 9970 & 0,0 \\
\hline PDT & 350 & 6,3 & 6095495 & 6,1 \\
\hline PHS & 13 & 0,2 & 324721 & 0,3 \\
\hline PMDB & 1199 & 21,6 & 18479511 & 18,5 \\
\hline PMN & 41 & 0,7 & 670982 & 0,6 \\
\hline PP & 553 & 9,9 & 6144206 & 6,2 \\
\hline PPS & 130 & 2,3 & 2813975 & 2,8 \\
\hline PR & 383 & 6,9 & 4274967 & 4,3 \\
\hline PRB & 54 & 1,0 & 1521641 & 1,5 \\
\hline PRP & 16 & 0,2 & 187674 & 0,2 \\
\hline PRTB & 11 & 0,2 & 197386 & 0,2 \\
\hline PSB & 312 & 5,6 & 5685266 & 5,7 \\
\hline PSG & 57 & 1,0 & 1019341 & 1,0 \\
\hline PSDB & 788 & 14,2 & 14445415 & 14,5 \\
\hline PSDG & 8 & 0,1 & 241561 & 0,2 \\
\hline PSL & 15 & 0,3 & 198247 & 0,2 \\
\hline PSOL & 0 & 0,0 & 795275 & 0,8 \\
\hline PSTU & 0 & 0,0 & 75573 & 0,1 \\
\hline PT & 554 & 10,0 & 16529237 & 16,6 \\
\hline PT do B & 8 & 0,1 & 214605 & 0,2 \\
\hline РТВ & 412 & 7,4 & 5066999 & 5,0 \\
\hline PTC & 13 & 0,2 & 190511 & 0,2 \\
\hline PTN & 16 & 0,3 & 182669 & 0,2 \\
\hline PV & 76 & 1,4 & 2967047 & 2,9 \\
\hline
\end{tabular}

Source : Ibid. 
Annexe II. - Maires Élus dans les capitales aux PREmier et SEcond tour DE L'ÉLEGTION MUNIGIPALE DE 2008

\begin{tabular}{|c|c|c|c|}
\hline Capitales de région & Élus au premier tour* & Élus au second tour* & Maires sortant réélus* \\
\hline \multicolumn{4}{|c|}{ Nord } \\
\hline Belem (PA) & & Dulcimar Costa (PTB) & Oui \\
\hline Boa vista ( $R R$ ) & $\begin{array}{l}\text { Iradilson Sampaio } \\
\text { (PSB) }\end{array}$ & & Oui \\
\hline Manaus (AM) & $\begin{array}{l}\text { Amazinho Mendes } \\
\text { (PTB) }\end{array}$ & & Oui \\
\hline Macapa (AP) & & Roberto Goes (PDT) & Non \\
\hline Palmas ( TO) & Raul Filho (PT) & & Oui \\
\hline Porto Velho & Roberto Sobrinho (PT) & & Oui \\
\hline Rio Branco (AG) & Angelim (PT) & & Oui \\
\hline \multicolumn{4}{|c|}{ Nordeste } \\
\hline Aracaju (SE) & $\begin{array}{l}\text { Edvaldo Nogueira } \\
\text { (PCdoB) }\end{array}$ & & Oui \\
\hline Fortaleza $(\mathrm{CE})$ & Luizianna Lins $(\mathrm{PT})$ & & Oui \\
\hline Joao Pessoa (PB) & Ricardo Coutinho (PSB) & & Oui \\
\hline Maceio (AL) & Cicero Almeida (PP) & & Oui \\
\hline Natal (RN) & Micarla de Souza (PV) & & Non \\
\hline Recife (PE) & João da Costa (PT) & & Non \\
\hline São Luis (MA) & & João Castelo (PSDB) & Non \\
\hline Teresina $(\mathrm{PI})$ & Silvio Mendes (PSDB) & & Oui \\
\hline Salvador(BA) & & João (PMDB) & Oui \\
\hline
\end{tabular}

Centre-Ouest

\begin{tabular}{|c|c|c|c|}
\hline Campo Grande (MS) & Neslinho Trad (PMDB) & & Oui \\
\hline Guiaba ( MT) & & Wilson Santos (PSDB) & Oui \\
\hline Goiania (GO) & Iris Rezende ( PMDB) & & Oui \\
\hline \multicolumn{4}{|c|}{ Sudeste } \\
\hline Belo Horizonte (MG) & & Marcio Lacerda (PSB) & Non \\
\hline Rio de Janeiro (RJ) & & Eduardo Paes (PMDB) & Non \\
\hline Sao Paulo (SP) & & Gilberto Kassab (DEM) & Oui \\
\hline Vitoria (ES) & & João Coser (PT) & Oui \\
\hline \multicolumn{4}{|c|}{ Sud } \\
\hline Curitiba (PR) & Beto Richa (PSDB) & & Oui \\
\hline Florianopolis (SG) & & Diario (PMDB) & Oui \\
\hline Porto Alegre (RS) & & José Fogaça (PMDB) & Oui \\
\hline
\end{tabular}

Note : Sont utilisés ici les noms d'usages des candidats. Au Brésil, les hommes et femmes politiques n'utilisent souvent que leur prénom pour se présenter.

Source: Ibid. 